Ces enseignements de langues sont complétés par des conférences d'ethnologie et d'histoire de l'Afrique, appelées à se développer dans l'avenir.

La création d'une nouvelle Section, 'Langues de l'Afrique', a également été décidée. Sont à l'étude dans ce cadre: le rétablissement des cours de Mandingue, de Haoussa, de Wolof et l'introduction de nouvelles langues négro-africaines au programme de l'École.

L'enseignement africaniste déjà donné à l'École (arabe, berbère, civilisation maghrébine, amharique, langues couchitiques, Histoire et Civilisations de l'Afrique Orientale) s'étendra ainsi à l'ensemble du continent.

(Communication de M. P. Alexandre)

\title{
Centre des Sciences Humaines de la République de Côte d'lvoire
}

En poursuivant la réorganisation de la recherche, le Gouvernement de la République de Côte d'Ivoire a décidé une transformation de l'ancien I.F.A.N. C'est ainsi qu'il a été créé, à compter du rer janvier 1960, un Centre des Sciences Humaines. Ce nouvel institut scientifique est placé sous la haute compétence du Ministre de l'Éducation nationale de Côte d'Ivoire.

Parmi les tâches principales du C.S.H. figure notamment celle d'organiser et de développer en Côte d'Ivoire toute recherche intéressant l'ethnologie, la sociologie, la démographie, l'économie, l'anthropologie physique, la préhistoire, l'archéologie, la linguistique, la phonétique, la musicologie, le folklore et la littérature orale, ainsi que l'étude des arts en général.

Le C.S.H. assume en outre la direction du Musée de la Côte d'Ivoire, la conservation et l'étude de ses riches collections dont le nombre atteint aujourd'hui environ 24.000 spécimens. Il est aussi chargé, par des échanges culturels internationaux, de contribuer à la connaissance dans le monde des civilisations de la Côte d'Ivoire. Enfin, il est appelé à assumer la mise'en route et la rédaction des publications scientifiques à tous les niveaux de compréhension.

Ce programme, prévu pour les années à venir, sera réalisé progressivement, conformément au plan d'extension prévu par le Ministère de l'Éducation nationale. M. B. Holas a été nommé Directeur du Centre des Sciences Humaines et, en cette qualité, Conservateur du Musée de la Côte d'Ivoire à Abidjan.

(Communication de M. B. Holas)

\section{La Mission Berliet-Ténéré}

10.000 kms. parcourus, dont 5.000 de Djanet à Djanet et 2.000 dans le désert du Ténéré situé au nord-ouest du lac Tchad, tel est l'exploit réalisé par les 9 camions ' Gazelle ' Berliet et 7 Land-Rovers d'accompagnement, de novembre 1959 à fin janvier 1960.

Le but de la mission était triple: faire des essais de camions spécialement adaptés au Sahara; rechercher des itinéraires transsahariens pour camions entre le Sud algérien, pays du pétrole, et les savanes soudano-tchadiennes, productrices de viande; faire l'etude scientifique des pays traversés.

Le premier but a été parfaitement atteint: le matériel s'est parfaitement bien comporté sur le long trajet Alger - Fort Lamy - Alger, à travers des pays difficiles: les grands ergs du Ténéré ont été l'obstacle majeur. Le second but, qui n'était d'ailleurs que subsidiaire, n'a été que partiellement atteint: l'itinéraire ouest Djanet - Arbre du Ténéré - Nguigmi et celui de l'est, Djanet - Bilma - Nguigmi offrent, à cause des ergs, de grandes difficultés. Il faudra probablement chercher ailleurs une voie plus commode.

Quant au troisième but, il fut rempli de façon satisfaisante. Géologues, préhistoriens, botaniste, zoologue, appartenant soit à l'Institut de Recherches Sahariennes d'Alger ou à l'Institut Français d'Afrique Noire à Dakar, firent ample moisson de matériel et d'observa- 
tions. Des mesures sur le magnétisme terrestre furent faites sur une bonne partie de l'itinéraire; des récoltes de préhistoire et d'échantillons de paléosols, de sables et de charbons permettront de résoudre certaines questions concernant le passé lointain de cette partie du Sahara et un herbier de 300 numéros fut rapporté.

Beaucoup reste encore à faire dans ce secteur peu accessible, au point de vue scientifique, mais cette mission aura permis, aux préhistoriens en particulier, de reconnaître les principaux points qui méritent une étude ultérieure plus détaillée.

Cette collaboration de l'industrie et de la science - chose nouvelle au Sahara - s'est donc avérée des plus fécondes et il est à souhaiter qu'elle se poursuive dans l'avenir.

(Communication du Dr. R. Mauny)

\section{Columbia University: West African Languages Survey}

The Ford Foundation has recently granted to Columbia University approximately $\$ 180,000$ for a 'West African Language Survey', a three-year project sponsored by a number of educational and research institutions in West Africa.

The objects of the project include substantive research in the indigenous languages of West Africa, the strengthening of West African institutions in the areas of general and African linguistics, and the training of Africans in linguistic research.

The programme includes field fellowships and grants-in-aid for linguistic research, and funds for the publication of linguistic materials concerning the West African area. The Director of the project is Professor Joseph H. Greenberg of Columbia University.

\section{(Communicated by Professor Joseph H. Greenberg)}

\section{Symposium on African Affairs at Lafayette College}

A symposium on the relevance to modern Africa of the revolutionary experience of the United States was held at Lafayette College, Easton, Pennsylvania, on ro and I I March. The principal speakers were Professor Gwendolen Carter, Mr. H. V. H. Sekyi, of the Ghana Embassy, Dr. Harry Rudin, Dr. Elliot Skinner, Dr. Eduardo Mondlane, Dr. Emory Ross, and Dr. Broadus Mitchell.

\section{L'Association Internationale de Droit Africain: la Section Belge}

CRÉÉE à Bruxelles dans les derniers mois de l'année I959, la section belge de cette Association a, depuis lors, poursuivi la tâche qui était la sienne: préparer la coopération avec les États d'Afrique et plus particulièrement le Congo belge en vue d'aider ces pays à résoudre les problèmes qui se présentent à eux dans le domaine du droit. Dans l'immédiat, la section belge s'était assignée deux objectifs: d'une part la création de sections indépendantes de l'Association en Afrique belge, d'autre part la préparation, en Belgique, d'un organe permanent à même d'assurer de manière efficace les tâches de coopération dans le domaine du droit. Il est déjà permis d'espérer la création prochaine de nouvelles sections à Coquilhatville, Élisabethville et Léopoldville ainsi qu'au Ruanda-Urundi. D'autre part la section belge multiplie les contacts afin que des sections soient aussi établies dans les autres provinces du Congo.

Outre ce premier objectif, la section belge souhaitait pouvoir constituer en Belgique un organisme permanent qui aurait pour tâche de centraliser, afin d'y répondre le plus rapidement possible, les demandes éventuelles de coopération émanant d'États africains. En outre, cet organisme devrait constituer, pour la première fois en Belgique, un véritable centre d'études du droit africain. Des contacts ont été, d'ores et déjà, pris avec le Ministère de l'Instruction Publique et on peut dire que la constitution de ce centre est en bonne voie. 